\title{
The interval between onset and admission predicts disease progression in COVID-19 patients
}

\author{
Liang Peng ${ }^{1 *} \wedge$, Qing-Quan Lv $^{2 *}$, Fan Yang ${ }^{3 \#}$, Xing-Mei Wu ${ }^{1}$, Cheng-Cheng Zhang ${ }^{1}$, Yong-Quan Wang ${ }^{1}$, \\ Wen-Hao Huang ${ }^{1}$, Chun-Wei Li ${ }^{1}$, Yi Wei ${ }^{1}$, Ren-Qiang Ma ${ }^{1}$, Ke-Jing Tang ${ }^{4}$, Lin Yao ${ }^{5}$, Jian Li ${ }^{1}$, Wei-Ping Wen ${ }^{1}$ \\ ${ }^{1}$ Department of Otorhinolaryngology, The First Affiliated Hospital of Sun Yat-sen University; International Airway Research Center, The First \\ Affiliated Hospital of Sun Yat-sen University, Guangzhou Key Laboratory of Otorhinolaryngology, Guangzhou, China; ${ }^{2}$ Department of Medical \\ Affairs, Hankou Hospital of Wuhan, Wuhan, China; ${ }^{3}$ Department of Anesthesiology, Hankou Hospital of Wuhan, Wuhan, China; ${ }^{4}$ Department \\ of Respiratory Medicine, The First Affiliated Hospital of Sun Yat-sen University, Guangzhou, China; ${ }^{5}$ Department of Medical Affairs, The Sixth \\ Affiliated Hospital of Sun Yat-sen University, Guangzhou, China
}

Contributions: (I) Conception and design: L Peng, QQ Lv, F Yang, L Yao, J Li, WP Wen; (II) Administrative support: L Yao, J Li, WP Wen; (III) Provision of study materials or patients: QQ Lv, F Yang, RQ Ma, L Yao; (IV) Collection and assembly of data: L Peng, QQ Lv, F Yang, XM Wu, CC Zhang, YQ Wang, WH Huang; (V) Data analysis and interpretation: All authors; (VI) Manuscript writing: All authors; (VII) Final approval of manuscript: All authors.

"These authors contributed equally to this work.

Correspondence to: Wei-Ping Wen. Department of Otorhinolaryngology, The First Affiliated Hospital, Sun Yat-sen University, No. 58 Zhongshan $2^{\text {nd }}$ Road, Guangzhou 510080, China. Email: wenwp@mail.sysu.edu.cn; Jian Li. Department of Otorhinolaryngology, The First Affiliated Hospital, Sun Yat-sen University, No. 58 Zhongshan $2^{\text {nd }}$ Road, Guangzhou 510080, China. Email: lijianent@hotmail.com; Lin Yao. Department of Medical Affairs, The Sixth Affiliated Hospital of Sun Yat-sen University, No. 26 Erheng Road, Yuancun, Guangzhou 510655, China.

Email: yaolin@mail.sysu.edu.cn.

Background: The prognostic role of the interval between disease onset and hospital admission (O-A interval) was undetermined in patients with the coronavirus disease 2019 (COVID-19).

Methods: A total of 205 laboratory-confirmed inpatients admitted to Hankou hospital of Wuhan from January 11 to March 8, 2020 were consecutively included in this retrospective observational study. Demographic data, medical history, laboratory testing results were collected from medical records. Univariate and multivariate logistic regression models were used to evaluate the prognostic effect of the O-A interval ( $\leq 7$ versus $>7$ days) on disease progression in mild-to-moderate patients. For severe-to-critical patients, the in-hospital mortality and the length of hospital stay were compared between the O-A interval subgroups using log-rank test and Mann-Whitney $U$ test, respectively.

Results: Mild-to-moderate patients with a short O-A interval ( $\leq 7$ days) are more likely to deteriorate to severe-to-critical stage compared to those with a long O-A interval ( $>7$ days) [unadjusted odds ratio $=2.93$, 95\% confidence interval (CI), 1.32-6.55; adjusted odds ratio $=3.44,95 \%$ CI, $1.20-9.83]$. No association was identified between the O-A interval and the mortality or the length of hospital stay of severe-to-critical patients. Conclusions: The O-A interval has predictive values for the disease progression in mild-to-moderate COVID-19 patients. Under circumstances of the specific health system in Wuhan, China, the spontaneous healthcare-seeking behavior is usually determined by patients' own heath conditions. Hence, the O-A interval can be reflective of the natural course of COVID-19 to some extent. However, our findings should be validated further in other cohorts and in other health systems.

Keywords: Coronavirus disease 2019 (COVID-19); prognosis; interval; health system

Submitted Jul 14, 2020. Accepted for publication Oct 30, 2020.

doi: $10.21037 /$ atm-20-5320

View this article at: http://dx.doi.org/10.21037/atm-20-5320

\footnotetext{
^ ORCID: 0000-0003-1682-7064.
} 


\section{Introduction}

The coronavirus disease 2019 (COVID-19) caused by the infection of severe acute respiratory syndrome coronavirus 2 (SARS-CoV-2) has rapidly spread around the world. As of June 24, 2020, the World Health Organization reported a total of 9,110,186 COVID-19 cases globally, with crude mortality of $5.19 \%$.

The clinical spectrum of SARS-CoV-2 infection ranges from asymptomatic infection and mild upper respiratory tract illness to severe pneumonia with respiratory failure and even death (1). Early detection of patients who are likely to develop severe illness is important and can help clinicians to optimize use of limited resources. Many predictive factors for severity of COVID-19 have been investigated and reported, including age, comorbidities (for example, hypertension, diabetes), laboratory test results, radiological findings etc. (2-12).

The COVID-19 crisis is an unprecedented challenge to health systems worldwide. Every country tries to find their own ways to fight against the pandemic. In China, the health system and government devote enormous resources to finding and curing every COVID-19 patient to effectively contain the epidemic. Suspected COVID-19 patient with or without symptoms can go to see a doctor at fever clinics of a tertiary hospital and get admitted to the designated hospital for COVID-19 if the COVID-19 diagnosis is confirmed. In United Kingdom, suspected COVID-19 patients with symptoms are usually advised to self-isolate at home and not to seek medical attention from National Health Service (NHS) until they cannot cope with their symptoms or their conditions get worse. These measures are useful to contain the epidemic and relieve the pressure of NHS. However, these measures may also lead to delays in patients receiving treatments to some extent. Wu et al. reported that early antiviral treatments may alleviate the severity and improve the prognosis of COVID-19 patients (13). Whether and how the interval between disease onset and hospital admission impacts on the prognosis of COVID-19 patients is worthy of investigation.

In current retrospective observational study, we aim to investigate the prognostic role of the interval between onset and admission (O-A interval) in COVID-19 patients. We present the following article in accordance with the STROBE reporting checklist (available at http://dx.doi. org/10.21037/atm-20-5320).

\section{Methods}

\section{Participants}

COVID-19 inpatients admitted to Hankou hospital of Wuhan (a tertiary designated hospital for COVID-19, reinforced by the medical stuff from hospitals of Sun Yat-sen University) from January 11, 2020 to March 8, 2020 were consecutively included in this study. COVID-19 diagnoses were confirmed by positive real-time reverse-transcription polymerase chain reaction (RT-PCR) assay for nasal or pharyngeal swab specimens as previously reported (1). Patients without laboratory-confirmed evidences of SARS$\mathrm{CoV}-2$ infection were excluded from the study. The study was conducted in accordance with the Declaration of Helsinki (as revised in 2013). This study was approved by the Institutional Review Board of the First Affiliated Hospital of Sun Yat-sen University (approval number: 2020-128); written informed consent was waived owing to the use of unidentified retrospective data.

\section{Data collection}

Demographic data, medical history, laboratory testing results, and medication treatments of included patients were collected from electronic medical records using a predefined form independently by two clinicians. Specially, the O-A interval was defined as the time from initial symptoms (mainly fever, cough, fatigue) onset to the date of admission, which could be extracted from the chief complains and the history of present illness in medical records. Disease severity on admission and the most severe form of the disease during the hospitalization of each patient were evaluated independently by two experienced clinicians by reviewing the medical records, and any disagreement was resolved by discussion to achieve a consensus. Mild cases were defined as patients with mild clinical symptoms and no sign of pneumonia on computer tomography imaging. Moderate cases were defined as patients with fever or/and respiratory tract symptoms and manifestations of pneumonia on computer tomography imaging. Severe cases were defined as patients met with any of the following criteria: (I) shortness of breath, respiration rate $\geq 30$ times/min; (II) the oxygen saturation measured by pulse oximetry $\leq 93 \%$ in resting state; (III) the ratio of arterial partial pressure of oxygen to fraction of inspiration oxygen 
$\leq 300 \mathrm{mmHg}$. Critical cases were defined as patients met with any of the following criteria: (I) respiratory failure, in need of mechanical ventilation; (II) shock; (III) other organ failure, admission to the intensive care unit. Data cut-off date was March 15, 2020.

\section{Outcomes}

For patients with mild-to-moderate disease on admission, the outcome was defined as disease deteriorating to severeto-critical stage during hospitalization. For patients with severe-to-critical disease on admission or during hospitalization, two types of outcomes were evaluated: death during hospitalization (time-to-event data) and length of hospital stay.

\section{Statistical analysis}

Normal distributed variables were displayed as mean \pm standard deviation and compared by independent-samples $t$-test. Non-normal distributed continuous variables were displayed as median (interquartile range) and compared by Mann-Whitney $\mathrm{U}$ test. Categorical variables were displayed as frequency (percentage) and compared by Pearson $\chi^{2}$ test or Fisher's exact test. Univariate and multivariate logistic regression models were used to evaluate the role of the O-A interval in predicting disease progression. Log-rank test was used to compare the mortality rates of severe-to-critical patients with different $\mathrm{O}-\mathrm{A}$ intervals. A two-sided $\mathrm{P}$ value $<0.05$ was considered statistically significant. Statistical analyses were conducted using Stata 14.1 (StataCorp).

\section{Results}

\section{Characteristics of patients}

A total of 205 patients were included in this study with a mean age of 58.4 years old. Male patients accounted for $47.8 \%$ of the cohort. Among the whole cohort, 118 patients were classified as mild-to-moderate cases on admission. Detailed demographic characteristics, laboratory findings on admission, and medication treatments are summarized in Table 1. The variables, C-reactive protein (CRP) and D-dimer, have the highest missing data rates, which are $31.7 \%$ and $24.4 \%$, respectively. Patients with severe-tocritical disease on admission seem to have higher neutrophil count, CRP, blood glucose, aspartate aminotransferase (AST), albumin, lactate dehydrogenase (LDH), fibrinogen, and D-dimer, shorter thrombin time (TT), and lower lymphocyte count compared to those with mild-tomoderate disease. The median of O-A interval of the whole cohort is 10 (interquartile range, 6-14) days, and the O-A interval is not associated with disease severity on admission. The patterns of disease progression are shown in Figure 1.

\section{Prognostic role of the $O$ - $A$ interval}

During the data extraction, we found that patients usually reported the time of symptoms onset using "week(s)" or "10 days" when the O-A interval was longer and they could not recall the date of onset exactly. Considering above, we defined the O-A interval $\leq 7$ days as short interval, while the O-A interval $>7$ days as long interval.

Of the 118 patients with mild-to-moderate disease on

Table 1 Characteristics of COVID-19 patients

\begin{tabular}{lcccc}
\hline \multirow{2}{*}{ Characteristics } & \multicolumn{3}{c}{ COVID-19 severity on admission } & \multirow{2}{*}{ P value } \\
\cline { 2 - 3 } Demographic & Total (N=205) & Mild-to-moderate (N=118) & Severe-to-critical (N=87) & \\
Age, year & $58.4 \pm 13.5$ & $57.2 \pm 13.5$ & $60.1 \pm 13.3$ & $0.131^{\mathrm{a}}$ \\
Male & $98(47.8 \%)$ & $56(47.5 \%)$ & $42(48.3 \%)$ & $0.908^{\mathrm{b}}$ \\
O-A interval, day & $10[6-14]$ & $10[6-14]$ & $10[7-14]$ & $0.270^{\mathrm{c}}$ \\
Comorbidity & & & & $0.336^{\mathrm{b}}$ \\
Any & $91(44.4 \%)$ & $49(41.5 \%)$ & $42(48.3 \%)$ & $0.522^{\mathrm{b}}$ \\
Diabetes & $36(17.6 \%)$ & $19(16.1 \%)$ & $17(19.5 \%)$ & $0.575^{\mathrm{b}}$ \\
Hypertension & $64(31.2 \%)$ & $35(29.7 \%)$ & $29(33.3 \%)$ &
\end{tabular}

Table 1 (continued) 
Table 1 (continued)

\begin{tabular}{|c|c|c|c|c|}
\hline Characteristics & \multicolumn{3}{|c|}{ COVID-19 severity on admission } & $P$ value \\
\hline CVD & $24(11.7 \%)$ & $12(10.2 \%)$ & $12(13.8 \%)$ & $0.425^{b}$ \\
\hline COPD & $8(3.9 \%)$ & $4(3.4 \%)$ & $4(4.6 \%)$ & $0.725^{\mathrm{d}}$ \\
\hline \multicolumn{5}{|l|}{ Laboratory findings on admission } \\
\hline Neutrophil count, $\times 10^{9} / \mathrm{L}$ & $3.5(2.4-5.4), n=196$ & $3.3(2.3-4.5), n=114$ & $4.1(2.5-6.9), \mathrm{n}=82$ & $0.036^{\mathrm{c}}$ \\
\hline Lymphocyte count, $\times 10^{9} / \mathrm{L}$ & $0.8(0.6-1.2), n=196$ & $1.0(0.7-1.4), n=114$ & $0.7(0.5-0.9), \mathrm{n}=82$ & $<0.001^{\mathrm{c}}$ \\
\hline Neutrophil-to-lymphocyte ratio & $4.13(2.17-8.00), n=196$ & 3.35 (1.89-5.93), $n=114$ & $5.13(2.85-11.85), n=82$ & $<0.001^{\mathrm{c}}$ \\
\hline Platelet count, $\times 10^{9} / \mathrm{L}$ & 194 [148-273], n=196 & 200 [155-275], n=114 & 174.5 [138-273], $n=82$ & $0.304^{c}$ \\
\hline Glucose, mmol/L & $6.08(5.11-8.25), n=188$ & 5.57 (4.95-7.31), $n=112$ & 6.89 (5.53-9.34), $n=76$ & $<0.001^{\mathrm{c}}$ \\
\hline Alanine aminotransferase, $U / L$ & 25 [17-40], n=194 & 24 [16-37], n=114 & $26[17-42], n=80$ & $0.199^{\circ}$ \\
\hline Aspartate aminotransferase, $\mathrm{U} / \mathrm{L}$ & 28 [20-40], n=194 & 27 [19-35], n=114 & $31[23-48], n=80$ & $0.005^{\mathrm{c}}$ \\
\hline Total bilirubin, mmol/L & $8.6(6.4-11.7), n=194$ & $8.5(6.4-10.5), n=114$ & $9.3(6.3-13.9), n=80$ & $0.122^{c}$ \\
\hline Albumin, g/L & $34.6(31.0-36.9), n=194$ & $35.0(31.8-37.7), n=114$ & $33.3(29.9-35.7), n=80$ & $0.008^{\mathrm{c}}$ \\
\hline Globulin, g/L & 29.7 (26.4-32.9), n=194 & 29.8 (25.9-33.0), n=114 & 29.5 (26.5-32.2), $n=80$ & $0.753^{\mathrm{c}}$ \\
\hline Blood urea nitrogen, $\mathrm{mmol} / \mathrm{L}$ & $4.58(3.39-5.85), n=190$ & 4.30 (3.27-5.49), $n=112$ & $5.00(3.53-7.05), n=78$ & $0.063^{c}$ \\
\hline Serum creatinine, $\mu \mathrm{mol} / \mathrm{L}$ & 65 [54-80], n=191 & 65 [54-78], n=113 & 70 [53-85], n=78 & $0.364^{\mathrm{c}}$ \\
\hline Fibrinogen, g/L & 3.65 (2.92-4.48), n=185 & $3.53(2.87-4.27), n=106$ & $4.06(3.02-4.62), n=79$ & $0.021^{\mathrm{c}}$ \\
\hline D-dimer, mg/L & $0.29(0.10-1.20), \mathrm{n}=155$ & $0.23(0.08-0.69), n=89$ & $0.54(0.14-1.77), n=66$ & $0.020^{c}$ \\
\hline \multicolumn{5}{|c|}{ Medication treatments during hospitalization } \\
\hline Antivirals & $71(34.6 \%)$ & $38(32.2 \%)$ & $33(37.9 \%)$ & $0.394^{b}$ \\
\hline Antibiotics & $180(87.8 \%)$ & $99(83.9 \%)$ & $81(93.1 \%)$ & $0.047^{\mathrm{b}}$ \\
\hline Intravenous steroids & $110(53.7 \%)$ & $53(44.9 \%)$ & $57(65.5 \%)$ & $0.003^{b}$ \\
\hline Intravenous immunoglobulin & $48(23.4 \%)$ & $24(20.3 \%)$ & $24(27.6 \%)$ & $0.226^{b}$ \\
\hline Traditional Chinses medicine & $56(27.3 \%)$ & $35(29.7 \%)$ & $21(24.1 \%)$ & $0.380^{\mathrm{b}}$ \\
\hline
\end{tabular}

All values are noted as mean \pm standard deviation, frequency (percentage) or median (interquartile range). $\mathrm{n}$ represents the number of patients with available data. O-A interval, the interval between onset and admission; CVD, cardiovascular or cerebrovascular disease; COPD, chronic obstructive pulmonary disease; WBC, white blood cell; APTT, activated partial thromboplastin time. ${ }^{a}$, independent-samples $t$-test; ${ }^{\text {b }}$, Pearson $\chi^{2}$ test; ${ }^{c}$, Mann-Whitney $\cup$ test; ${ }^{d}$, Fisher's exact test. 


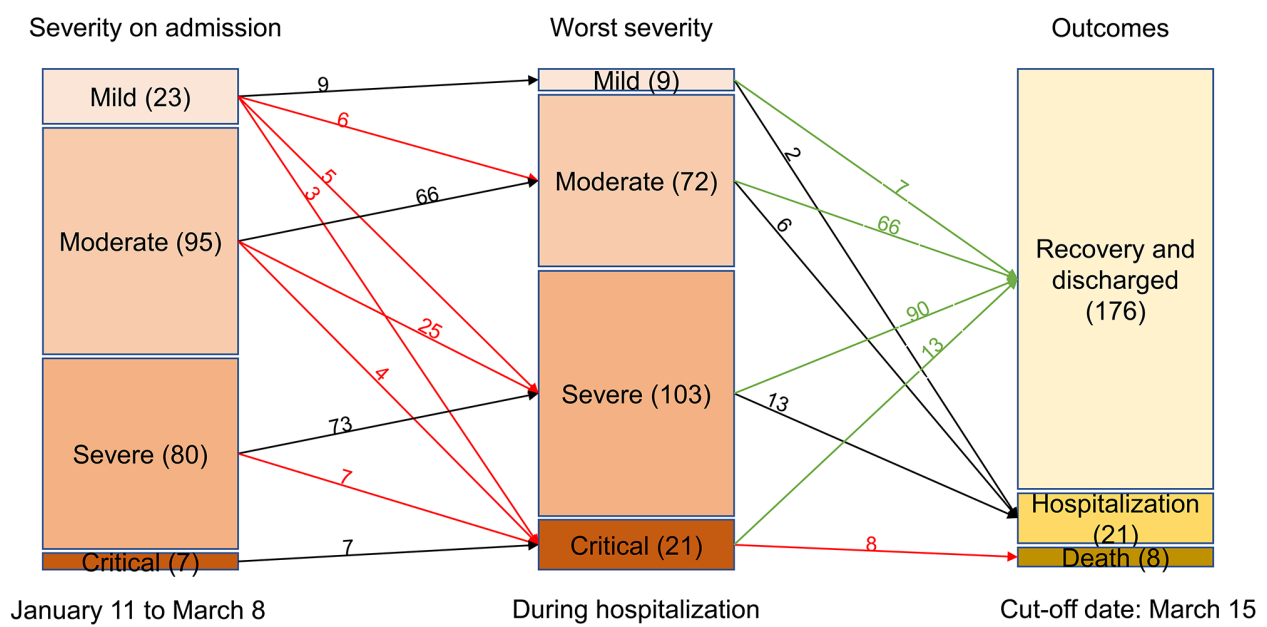

Figure 1 Overview of disease progression patterns in COVID-19 patients.

Table 2 Unadjusted and adjusted prognostic role of O-A interval on disease progression

\begin{tabular}{|c|c|c|c|c|}
\hline Variables & \multicolumn{2}{|c|}{ Univariate regression } & \multicolumn{2}{|c|}{ Multivariate regression } \\
\hline O-A interval $(\leq 7$ vs. $>7 \mathrm{~d})$ & $2.93(1.32-6.55)$ & 0.009 & $3.44(1.20-9.83)$ & 0.021 \\
\hline Age (>60 vs. $\leq 60 \mathrm{y})$ & $5.00(2.15-11.64)$ & $<0.001$ & $2.22(0.72-6.87)$ & 0.166 \\
\hline Comorbidity (any vs. none) & 3.48 (1.54-7.83) & 0.003 & $2.80(0.88-8.91)$ & 0.081 \\
\hline Thrombin time, s & $1.11(0.94-1.31)$ & 0.206 & & \\
\hline Total bilirubin, $\mathrm{mmol} / \mathrm{L}$ & $1.08(0.97-1.20)$ & 0.170 & & \\
\hline Albumin, g/L & $0.93(0.85-1.00)$ & 0.061 & & \\
\hline Blood urea nitrogen, $\mathrm{mmol} / \mathrm{L}$ & $1.28(1.06-1.55)$ & 0.010 & $1.31(0.93-1.83)$ & 0.120 \\
\hline
\end{tabular}

Complete-case analysis was adopted in multivariate logistic regression model. 106/118 cases were included in multivariate analysis, and 34/106 cases experienced disease deterioration. O-A interval, the interval between onset and admission; OR, odds ration; Cl, confidence interval.

admission, 37 patients deteriorated to severe-to-critical stage during hospitalization (Figure 1). The characteristics of mild-to-moderate patients with different O-A intervals are summarized in Table S1. Univariate logistic regression analysis revealed that the short $\mathrm{O}-\mathrm{A}$ interval is associated with higher risk of disease progression [odds ratio (OR) $=2.93,95 \%$ CI, 1.32-6.55] (Table 2). After reviewing previous studies (2-10) and consultation with experienced clinicians, we select age ( $>60 v s . \leq 60 \mathrm{y})$, comorbidities (including hypertension, diabetes, cardiovascular or cerebrovascular disease, and chronic obstructive pulmonary disease), neutrophil-to-lymphocyte ratio (NLR), CRP, D-dimer, total bilirubin, albumin, blood urea nitrogen (BUN), LDH as potential covariates to adjust the effect of the $\mathrm{O}-\mathrm{A}$ interval in a multivariate logistic regression model. However, CRP and D-dimer are ruled out because of the high proportion of missing data. Among other coagulation indexes (prothrombin time, international normalized ratio, activated partial thromboplastin time, TT, fibrinogen), we choose TT to replace D-dimer as TT is most closely correlated with D-dimer (Table S2). Considering the limited sample size, only covariates with statistically significant 
A

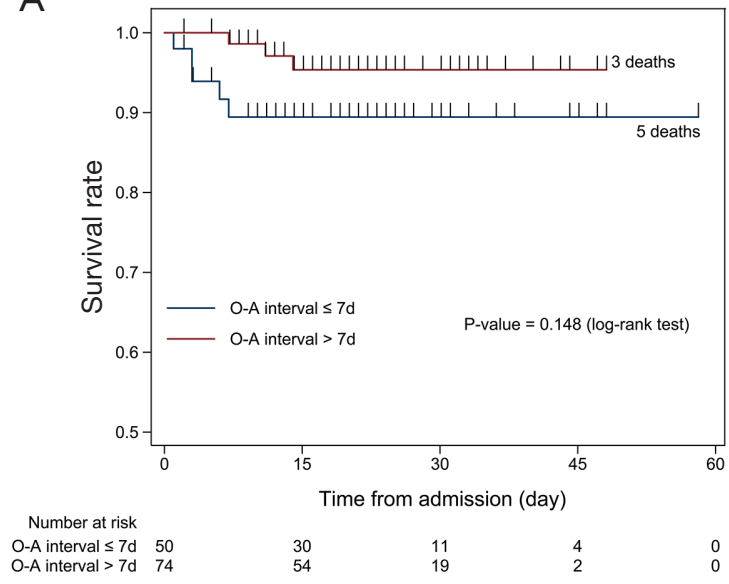

B

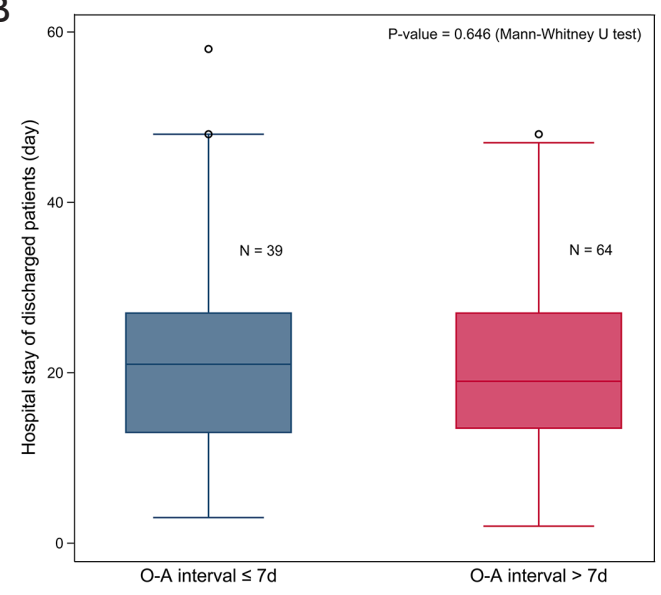

Figure 2 Prognostic role of the O-A interval in severe-to-critical patients. (A) Kaplan-Meier curves depicting mortality during hospitalization. (B) Boxplots depicting the length of hospital stay of discharged patients. O-A interval, interval between onset and admission.

prognostic effects in the univariate logistic regression model ( $\mathrm{P}$ value $<0.05$ ) can enter the multivariate logistic regression model. Finally, age, comorbidities, NLR, LDH, and BUN entered the multivariate logistic regression model as covariates. After adjustment, the short O-A interval is still associated with higher risk of disease progression (OR =3.44, 95\% CI, 1.20-9.83) (Table 2).

Of 124 patients with severe-to-critical disease on admission or during hospitalization, 8 patients died, 103 patients recovered and got discharged, and 13 patients were still in hospital by the data cut-off date (Figure 1). We did not find prognostic effects of the O-A interval on death rates of severe-to-critical patients during hospitalization (Figure 2A). Besides, we did not find impacts of the O-A interval on the length of hospital stay of discharged patients (Figure 2B).

\section{Discussion}

In this observational study, we investigated the potential prognostic effect of the O-A interval in COVID-19 inpatients. We should understand what the O-A interval stands for before we can interpret the prognostic role of the O-A interval. In China, the hierarchical medical system is less developed, and citizens who feel unwell can go to see a doctor in a tertiary hospital directly without a reference from general physicians. If necessary, patients can be admitted to hospital by outpatient doctors. During the epidemic in Wuhan, tens of thousands of healthcare workers from other part of China reinforced the medical system of Wuhan to make sure that every suspected case was tested, and every confirmed case was treated properly. Under such circumstances in Wuhan, the spontaneous healthcare-seeking behavior is usually determined by patients' own heath conditions. In addition, we found that the disease severity on admission was not associated with the O-A interval. Hence, the O-A interval is reflective of the time by which COVID-19 develops into a stage when patients find it necessary to go to hospital.

To predict the prognosis of COVID-19 patients is to predict how the virus and host interact with each other. Symptoms, signs, laboratory test results, and imagining features can reflect the interaction between the virus and host partially, and can be of prognostic roles, as previously reported (2-10). In this study, we found that the O-A interval could predict disease progression during hospitalization in mild-to-moderate patients. Patients with the O-A interval $\leq 7$ days could remember the date of initial symptoms onset precisely indicating that these initial symptoms were usually typical and impressive. The shorter O-A interval which indicates rapid symptoms worsening, may also reflect the nature of interaction between the virus and host. This may explain why mild-tomoderate COVID-19 patients with shorter O-A interval are more likely to deteriorate during hospitalization. The O-A interval can be accessed from history taking easily on admission. This predictor can give clinicians quick impression about disease progression in mild-to-moderate patients and aid in making clinical decisions to some extent.

On the other hand, the O-A interval does not seem to 
influence the mortality in patients with severe-to-critical disease. However, we should note that the statistical power in mortality analysis was low due to the small event number. We also compared the length of hospital stay between discharged severe-to-critical patients with a short O-A interval and those with a long O-A interval and found no difference. It seems that the O-A interval has no prognostic role in severe-to-critical patients.

When we try to interpret the result in this study, we should bear in mind that the O-A interval reflects the nature course of COVID-19 under the circumstances of Chinese strategy against epidemic. In different countries or regions with different health systems adopting different anti-epidemic strategies, the O-A interval may have different meanings, and hence have different prognostic values. For example, in United Kingdom, people with mild diseases are encouraged to self-isolate at home and not to seek medical attention from NHS until they cannot cope with their symptoms or their conditions get worse. Under this circumstance, the O-A interval may be prognostic for patients who are present in the urgent care or emergency room and usually with severe form of COVID-19, which can help healthcare providers at those situations decide the disposition of the patients. However, whether the longer $\mathrm{O}-\mathrm{A}$ interval caused by delay in receiving treatments would influence the prognosis of COVID-19 patients or not still needs to be further investigated by cross-regional studies in the future.

Some limitations of this study should be noted. First, the statistical power was limited by the small sample size of this study. Our findings should be validated in a larger cohort in other institutions in the future. Second, due to the retrospective study design, not all laboratory tests were done in all patients. Selection bias may be caused by excluding cases with missing data from analyses. Third, not all potential confounding factors were considered in multivariate analysis due to the limited sample size. CRP and D-dimer which are important prognostic factors in COVID-19 (8,14-17), were not included in the multivariate analysis due to the high proportion of missing data. However, in supplementary multivariate analyses including CRP or D-dimer as one of the covariates, the O-A interval remained to be an independent prognostic factor in mildto-moderate patients (Tables S3,S4).

\section{Conclusions}

The O-A interval can predict disease progression in
COVID-19 patients. Mild-to-moderate patients with a short O-A interval ( $\leq 7$ days) are more likely to deteriorate to severe-to-critical stage. Our findings should be validated in other cohorts and in other health systems.

\section{Acknowledgments}

We are extremely thankful to the medical stuff from hospitals of Sun Yat-sen University who volunteered to reinforce Wuhan during the critical time.

Funding: This work was supported by the Science and Technology Planning Project of Guangdong Province (No. 2020B1111300003) and the 2020 Key Scientific Emergency Project for New Coronavirus Prevention and Control of Sun Yat-sen University [80000-18843416].

\section{Footnote}

Reporting Checklist: The authors have completed the STROBE reporting checklist. Available at http://dx.doi. org/10.21037/atm-20-5320

Data Sharing Statement: Available at http://dx.doi. org/10.21037/atm-20-5320

Peer Review File: Available at http://dx.doi.org/10.21037/ atm-20-5320

Conflicts of Interest: All authors have completed the ICMJE uniform disclosure form (available at http://dx.doi. org/10.21037/atm-20-5320). The authors have no conflicts of interest to declare.

Ethical Statement: The authors are accountable for all aspects of the work in ensuring that questions related to the accuracy or integrity of any part of the work are appropriately investigated and resolved. The study was conducted in accordance with the Declaration of Helsinki (as revised in 2013). This study was approved by the Institutional Review Board of the First Affiliated Hospital of Sun Yat-sen University (approval number: 2020-128); written informed consent was waived owing to the use of unidentified retrospective data.

Open Access Statement: This is an Open Access article distributed in accordance with the Creative Commons Attribution-NonCommercial-NoDerivs 4.0 International License (CC BY-NC-ND 4.0), which permits the non- 
commercial replication and distribution of the article with the strict proviso that no changes or edits are made and the original work is properly cited (including links to both the formal publication through the relevant DOI and the license). See: https://creativecommons.org/licenses/by-nc-nd/4.0/.

\section{References}

1. Huang C, Wang Y, Li X, et al. Clinical features of patients infected with 2019 novel coronavirus in Wuhan, China. Lancet 2020;395:497-506.

2. Liang $\mathrm{W}$, Liang $\mathrm{H}$, Ou L, et al. Development and Validation of a Clinical Risk Score to Predict the Occurrence of Critical Illness in Hospitalized Patients With COVID-19. JAMA Intern Med 2020;180:1081-9.

3. Wu C, Chen X, Cai Y, et al. Risk Factors Associated With Acute Respiratory Distress Syndrome and Death in Patients With Coronavirus Disease 2019 Pneumonia in Wuhan, China. JAMA Intern Med 2020;180:934-43.

4. Gong J, Ou J, Qiu X, et al. A Tool to Early Predict Severe Corona Virus Disease 2019 (COVID-19) : A Multicenter Study using the Risk Nomogram in Wuhan and Guangdong, China. Clin Infect Dis 2020;71:833-40.

5. Liu W, Tao ZW, Wang L, et al. Analysis of factors associated with disease outcomes in hospitalized patients with 2019 novel coronavirus disease. Chin Med J (Engl) 2020;133:1032-8.

6. Li X, Xu S, Yu M, et al. Risk factors for severity and mortality in adult COVID-19 inpatients in Wuhan. J Allergy Clin Immunol 2020;146:110-8.

7. Liu Y, Du X, Chen J, et al. Neutrophil-to-lymphocyte ratio as an independent risk factor for mortality in hospitalized patients with COVID-19. J Infect

Cite this article as: Peng L, Lv QQ, Yang F, Wu XM, Zhang CC, Wang YQ, Huang WH, Li CW, Wei Y, Ma RQ, Tang KJ, Yao L, Li J, Wen WP. The interval between onset and admission predicts disease progression in COVID-19 patients. Ann Transl Med 2021;9(3):213. doi: 10.21037/atm-20-5320 2020;81:e6-e12.

8. Tan C, Huang Y, Shi F, et al. C-reactive protein correlates with computed tomographic findings and predicts severe COVID-19 early. J Med Virol 2020;92:856-62.

9. Zheng Z, Peng F, Xu B, et al. Risk factors of critical \& mortal COVID-19 cases: A systematic literature review and meta-analysis. J Infect 2020;81:e16-e25.

10. Wynants L, Van Calster B, Bonten MMJ, et al. Prediction models for diagnosis and prognosis of covid-19 infection: systematic review and critical appraisal. BMJ 2020;369:m1328.

11. Lippi G, Wong J, Henry BM. Hypertension in patients with coronavirus disease 2019 (COVID-19): a pooled analysis. Pol Arch Intern Med 2020;130:304-9.

12. Tadic M, Cuspidi C, Sala C. COVID-19 and diabetes: Is there enough evidence? J Clin Hypertens (Greenwich) 2020;22:943-8.

13. Wu J, Li W, Shi X, et al. Early antiviral treatment contributes to alleviate the severity and improve the prognosis of patients with novel coronavirus disease (COVID-19). J Intern Med 2020;288:128-38.

14. Liu F, Li L, Xu M, et al. Prognostic value of interleukin-6, C-reactive protein, and procalcitonin in patients with COVID-19. J Clin Virol 2020;127:104370.

15. Wang L. C-reactive protein levels in the early stage of COVID-19. Med Mal Infect 2020;50:332-4.

16. Zhang L, Yan X, Fan Q, et al. D-dimer levels on admission to predict in-hospital mortality in patients with Covid-19. J Thromb Haemost 2020;18:1324-9.

17. Li Y, Zhao K, Wei H, et al. Dynamic relationship between D-dimer and COVID-19 severity. Br J Haematol 2020;190:e24-7. 
Table S1 Characteristics of mild-to-moderate COVID-19 patients

\begin{tabular}{|c|c|c|c|c|}
\hline \multirow{2}{*}{ Characteristics } & \multicolumn{3}{|c|}{ Mild-to-moderate patients } & \multirow{2}{*}{ P-value } \\
\hline & Total $\mathrm{N}=118$ & O-A interval $\leq 7$ days $\mathrm{N}=49$ & O-A interval $>7$ days $\mathrm{N}=69$ & \\
\hline \multicolumn{5}{|l|}{ Demographic } \\
\hline Age, year & $57.2 \pm 13.5$ & $58.3 \pm 14.9$ & $56.4 \pm 12.5$ & $0.445^{\mathrm{a}}$ \\
\hline Male & $56(47.5 \%)$ & 27 (55.1\%) & $29(42.0 \%)$ & $0.161^{\mathrm{b}}$ \\
\hline \multicolumn{5}{|l|}{ Comorbidity } \\
\hline Any & 49 (41.5\%) & $20(40.8 \%)$ & $29(42.0 \%)$ & $0.895^{\mathrm{b}}$ \\
\hline Diabetes & $19(16.1 \%)$ & $10(20.4 \%)$ & $9(16.1 \%)$ & $0.283^{b}$ \\
\hline Hypertension & 35 (29.7\%) & $15(30.6 \%)$ & $20(29.0 \%)$ & $0.849^{b}$ \\
\hline CVD & $12(10.2 \%)$ & $5(10.2 \%)$ & $7(10.1 \%)$ & $1.000^{d}$ \\
\hline COPD & $4(3.4 \%)$ & $1(2.0 \%)$ & $3(4.3 \%)$ & $0.640^{d}$ \\
\hline \multicolumn{5}{|l|}{ Laboratory findings on admission } \\
\hline WBC count, $\times 10^{9} / \mathrm{L}$ & $4.6(3.7-6.0) n=114$ & $4.4(3.4-5.8) n=48$ & $5.0(3.9-6.4) n=66$ & $0.240^{\circ}$ \\
\hline Neutrophil count, $\times 10^{9} / \mathrm{L}$ & $3.3(2.3-4.5) n=114$ & $3.0(2.3-4.4) n=48$ & $3.6(2.3-4.6) n=66$ & $0.328^{\mathrm{c}}$ \\
\hline Lymphocyte count, $\times 10^{9} / \mathrm{L}$ & $1.0(0.7-1.4) n=114$ & $0.9(0.6-1.2) n=48$ & $1.1(0.7-1.5) n=66$ & $0.039^{\circ}$ \\
\hline Neutrophil-to-lymphocyte ratio & $3.35(1.89-5.93) n=114$ & $4.15(1.72-5.98) n=48$ & $2.70(1.91-5.85) n=66$ & $0.486^{c}$ \\
\hline Platelet count, $\times 10^{9} / \mathrm{L}$ & $200(155-275) n=114$ & $175(132-200) n=48$ & $236(190-302) n=66$ & $<0.001^{\circ}$ \\
\hline Hemoglobin, $g / L$ & $128(120-140) n=114$ & $132(121-143) n=48$ & $128(118-136) n=66$ & $0.173^{\mathrm{c}}$ \\
\hline C-reactive protein, $\mathrm{mg} / \mathrm{L}$ & $21.1(3.6-35.0) n=85$ & $30.7(16.4-36.0) n=33$ & $8.3(1.6-33.6) n=52$ & $0.004^{c}$ \\
\hline Glucose, $\mathrm{mmol} / \mathrm{L}$ & $5.57(4.95-7.31) n=112$ & $5.72(5.08-7.42) n=47$ & $5.34(4.86-7.05) n=65$ & $0.217^{\mathrm{c}}$ \\
\hline Alanine aminotransferase, $\mathrm{U} / \mathrm{L}$ & $24(16-37) n=114$ & $25(16-40) n=47$ & $22(16-36) n=67$ & $0.874^{\mathrm{c}}$ \\
\hline Aspartate aminotransferase, U/L & $27(19-35) n=114$ & $27(21-40) n=47$ & $23(17-30) n=67$ & $0.006^{c}$ \\
\hline Total bilirubin, $\mathrm{mmol} / \mathrm{L}$ & $8.5(6.4-10.5) n=114$ & $7.8(5.9-9.6) n=47$ & $8.8(6.8-11.4) n=67$ & $0.067^{c}$ \\
\hline Albumin, $g / L$ & $35.0(31.8-37.7) n=114$ & $35.2(32.1-38.3) n=47$ & $35.0(31.2-37.1) n=67$ & $0.232^{\mathrm{c}}$ \\
\hline Globulin, g/L & 29.8 (25.9-33.0), $n=114$ & $29.7(25.6-33.1) n=47$ & $29.8(25.9-32.9) n=67$ & $0.856^{\mathrm{c}}$ \\
\hline Blood urea nitrogen, $\mathrm{mmol} / \mathrm{L}$ & $4.30(3.27-5.49) n=112$ & $4.43(3.66-5.49) n=47$ & $4.07(3.09-5.57) n=65$ & $0.334^{\circ}$ \\
\hline Serum creatinine, $\mu \mathrm{mol} / \mathrm{L}$ & $65(54-78) n=113$ & $68(57-78) n=48$ & $62(52-79) n=65$ & $0.222^{c}$ \\
\hline Lactate dehydrogenase, IU/L & $218(180-285) n=112$ & $231(182-294) n=47$ & $208(180-280) n=65$ & $0.308^{\mathrm{c}}$ \\
\hline Prothrombin time, s & $13.9(13.1-15.6) n=106$ & $13.8(12.9-15.9) n=44$ & $13.9(13.2-15.1) n=62$ & $0.562^{\mathrm{c}}$ \\
\hline International normalized ratio & $1.1(1.03-1.17) n=106$ & $1.09(1.02-1.18) n=44$ & $1.10(1.05-1.17) n=62$ & $0.317^{\mathrm{c}}$ \\
\hline APTT, s & $34.8(31.1-37.0) n=106$ & $35.5(31.7-37.4) n=44$ & $34.3(31.0-36.4) n=62$ & $0.237^{\circ}$ \\
\hline Thrombin time, s & $15.4(14.7-16.0) n=106$ & $15.1(14.4-15.9) n=44$ & $15.6(15.0-16.2) n=62$ & $0.073^{\mathrm{c}}$ \\
\hline Fibrinogen, g/L & $3.53(2.87-4.27) n=106$ & $3.88(3.08-4.39) n=44$ & $3.34(2.64-4.10) n=62$ & $0.038^{\mathrm{c}}$ \\
\hline D-dimer, mg/L & $0.23(0.08-0.69) n=89$ & $0.15(0.05-0.75) n=39$ & $0.26(0.11-0.66) n=50$ & $0.284^{c}$ \\
\hline \multicolumn{5}{|c|}{ Medication treatments during hospitalization } \\
\hline Antivirals & $38(32.2 \%)$ & $15(30.6 \%)$ & $23(33.3 \%)$ & $0.755^{b}$ \\
\hline Antibiotics & 99 (83.9\%) & $46(93.9 \%)$ & $53(76.8 \%)$ & $0.013^{b}$ \\
\hline Intravenous steroids & $53(44.9 \%)$ & $27(55.1 \%)$ & $26(37.7 \%)$ & $0.061^{b}$ \\
\hline Intravenous immunoglobulin & $24(20.3 \%)$ & $10(20.4 \%)$ & $14(20.3 \%)$ & $0.987^{b}$ \\
\hline Traditional Chinses medicine & $35(29.7 \%)$ & $15(30.6 \%)$ & $20(29.0 \%)$ & $0.849^{b}$ \\
\hline \multicolumn{5}{|l|}{ Outcome } \\
\hline Deterioration & 37 (31.4\%) & $22(44.9 \%)$ & $15(21.7 \%)$ & $0.008^{\mathrm{b}}$ \\
\hline
\end{tabular}

All values are noted as mean \pm standard deviation, frequency (percentage) or median (interquartile range). $n$ represents the number of patients with available data. Abbreviations: O-A interval, the interval between onset and admission; CVD, cardiovascular or cerebrovascular disease; COPD, chronic obstructive pulmonary disease; WBC, white blood cell; APTT, activated partial thromboplastin time. a Independent-samples $t$ test. b Pearson $\chi^{2}$ test. c Mann-Whitney $U$ test. $d$ Fisher's exact test. 
Table S2 Correlation between D-dimer and other coagulation indexes

\begin{tabular}{lcc}
\hline Coagulation indexes & Correlation coefficient & P-value \\
\hline Prothrombin time & 0.053 & 0.518 \\
International normalized ratio & 0.068 & 0.403 \\
Activated partial thromboplastin time & -0.108 & 0.183 \\
Thrombin time & 0.417 & $<0.001$ \\
Fibrinogen & -0.257 & 0.001 \\
\hline
\end{tabular}

$\mathrm{N}=154$. Correlation between $\mathrm{D}$-dimer and other coagulation indexes was evaluated by Pearson correlation test.

Table S3 Multivariate logistic analyses including C-reactive protein as covariate

\begin{tabular}{|c|c|c|c|c|}
\hline Variables & \multicolumn{2}{|c|}{ Model $1^{a}$} & \multicolumn{2}{|c|}{ Model $2^{b}$} \\
\hline O-A interval $(\leq 7 \mathrm{~d}$ vs. $>7 \mathrm{~d})$ & $3.67(1.11-12.20)$ & 0.033 & $2.91(1.07-7.91)$ & 0.037 \\
\hline C-reactive protein, $\mathrm{mg} / \mathrm{L}$ & $1.01(0.97-1.06)$ & 0.517 & $1.05(1.01-1.08)$ & 0.008 \\
\hline Age $(>60$ y vs. $\leq 60$ y) & $1.43(0.37-5.50)$ & 0.600 & & \\
\hline Comorbidity (any vs. none) & $2.49(0.69-9.02)$ & 0.166 & & \\
\hline Blood urea nitrogen, $\mathrm{mmol} / \mathrm{L}$ & $1.19(0.82-1.73)$ & 0.363 & & \\
\hline Lactate dehydrogenase, IU/L & $1.01(1.00-1.01)$ & 0.090 & & \\
\hline
\end{tabular}

Abbreviations: O-A interval, the interval between onset and admission; OR, odds ratio; $\mathrm{Cl}$, confidence interval. a Model 1 includes 77 mild-to-moderate cases, of which 28 cases deteriorated. b Model 2 includes 85 mild-to-moderate cases, of which 30 cases deteriorated.

Table S4 Multivariate logistic analyses including D-dimer as covariate

\begin{tabular}{|c|c|c|c|c|}
\hline Variables & \multicolumn{2}{|c|}{ Model $1^{\text {a }}$} & \multicolumn{2}{|c|}{ Model $2^{b}$} \\
\hline O-A interval $(\leq 7 \mathrm{~d}$ vs. $>7 \mathrm{~d})$ & $5.33(1.39-20.42)$ & 0.015 & $4.30(1.60-11.54)$ & 0.004 \\
\hline D-dimer, mg/L & $1.02(0.73-1.42)$ & 0.910 & $1.28(1.03-1.59)$ & 0.024 \\
\hline Age $(>60$ y vs. $\leq 60$ y) & $1.62(0.35-7.48)$ & 0.537 & & \\
\hline Neutrophil-to-lymphocyte ratio & $1.03(0.90-1.18)$ & 0.668 & & \\
\hline Blood urea nitrogen, $\mathrm{mmol} / \mathrm{L}$ & $1.51(0.96-2.36)$ & 0.073 & & \\
\hline Lactate dehydrogenase, IU/L & $1.01(1.00-1.02)$ & 0.011 & & \\
\hline
\end{tabular}

Abbreviations: O-A interval, the interval between onset and admission; OR, odds ratio; $\mathrm{Cl}$, confidence interval. a Model 1 includes 83 mild-to-moderate cases, of which 27 cases deteriorated. b Model 2 includes 89 mild-to-moderate cases, of which 29 cases deteriorated. 